\title{
N-Octane Diffusivity Enhancement via Carbon Dioxide in Silica Slit-Shaped NanoPores - A Molecular Dynamics Simulation
}

\author{
Thu Le, Stephanie Ogbe and Alberto Striolo* \\ Department of Chemical Engineering, University College London, London WC1E 6BT \\ United Kingdom \\ David R. Cole \\ School of Earth Sciences, Ohio State University, Columbus, Ohio 43210 \\ United States
}

\begin{abstract}
Equilibrium molecular dynamics simulations were conducted to study the competitive adsorption and diffusion of mixtures containing n-octane and carbon dioxide confined in slitshaped silica pores of width $1.9 \mathrm{~nm}$. Atomic density profiles substantiate strong interactions between $\mathrm{CO}_{2}$ molecules and the protonated pore walls. Non-monotonic change in n-octane self-diffusion coefficients as a function of $\mathrm{CO}_{2}$ loading was observed. $\mathrm{CO}_{2}$ preferential adsorption to the pore surface is likely to attenuate the surface adsorption of n-octane, lower the activation energy for n-octane diffusivity, and consequently enhance n-octane mobility at low $\mathrm{CO}_{2}$ loading. This observation was confirmed by conducting test simulations for pure $\mathrm{n}$ octane confined in narrower pores. At high $\mathrm{CO}_{2}$ loading, n-octane diffusivity is hindered by molecular crowding. Thus, n-octane diffusivity displays a maximum. In contrast, within the concentration range considered here, the self-diffusion coefficient predicted for $\mathrm{CO}_{2}$ exhibits a monotonic increase with loading, which is attributed to a combination of effects including the saturation of the adsorption capacity of the silica surface. Test simulations suggest that the results are strongly dependent on the pore morphology, and in particular on the presence of edges that can preferentially adsorb $\mathrm{CO}_{2}$ molecules and therefore affect the distribution of these molecules equally on the pore surface, which is required to provide the effective enhancement of n-octane diffusivity.
\end{abstract}

* Author to whom correspondence should be addressed: a.striolo@ucl.ac.uk

Keywords: Mixture; Competitive Adsorption; Preferential Adsorption 


\section{INTRODUCTION}

The increasing concerns about global warming have prompted researchers to identify different strategies for long-term $\mathrm{CO}_{2}$ sequestration. A common approach is coupling $\mathrm{CO}_{2}$ sequestration with enhanced oil recovery, in which $\mathrm{CO}_{2}$ can be used as an additive for tertiary oil recovery in depleted petroleum reservoirs. ${ }^{1}$ It is desired that, once injected, $\mathrm{CO}_{2}$ displaces the hydrocarbons and undergoes mineralization to be permanently trapped within the rock formation. ${ }^{2}$ Understanding the molecular phenomena related to adsorption and transport of $\mathrm{CO}_{2}$-hydrocarbon mixtures in the sub-surface is therefore necessary in order to optimize both $\mathrm{CO}_{2}$ storage capacity, as well as oil and gas production. Despite the vast literature on binary $\mathrm{CO}_{2}$ and $\mathrm{CH}_{4}$ mixtures, ${ }^{3-5}$ few studies focused on adsorption and dynamics of $\mathrm{CO}_{2}$ mixtures with more economically attractive, higher molecular weight hydrocarbons under confinement. ${ }^{6,7}$

We previously conducted extensive molecular dynamics (MD) simulations to study propane adsorption, structure and diffusion in slit-shaped silica pores at sub-, near-, and super-critical conditions. ${ }^{8}$ The results were qualitatively consistent with the experimental adsorption isotherms reported by Gruszkiewicz et al., ${ }^{9}$ and the SANS data reported by Rother et al. ${ }^{10} \mathrm{We}$ recently conducted $\mathrm{MD}$ simulations for the structure and dynamics of $\mathrm{CO}_{2}$-butane mixtures confined within slit-shaped silica pores. ${ }^{11}$ Preferential adsorption of carbon dioxide near the $\mathrm{OH}$ groups on the surface was observed, where the adsorbed $\mathrm{CO}_{2}$ molecules tend to interact simultaneously with more than one $-\mathrm{OH}$ group. Analysis of the simulation results suggests that the preferential $\mathrm{CO}_{2}$ adsorption to the pore walls weakens the adsorption of n-butane, lowers the activation energy for n-butane diffusivity, and consequently enhances n-butane mobility. Building on these foundations and as an effort to complete our understanding about the system, in this work, we investigate the effect of increasing $\mathrm{CO}_{2}$ loading and the pore morphology on the mobility of all confined fluids. The same solid substrate, $\beta$-cristobalite, was used to compare the new results to those obtained previously. MD simulations are chosen for their ability to provide extensive insights regarding effective fluid-solids interactions, structural (i.e. density profile) and dynamic properties (i.e. mobility) of all components of interest.

In the next section, we provide detailed information about the simulation models and methodology. Next, results are presented and analyzed. Lastly, our conclusions are summarized. 


\section{SIMULATION MODELS AND METHODOLOGY}

Extensive MD simulations for binary mixtures of $\mathrm{CO}_{2} / \mathrm{n}-\mathrm{C}_{8} \mathrm{H}_{18}$ confined within slit-shaped silica pores were performed. As a proxy for materials often found in the sub-surface, the solid substrate considered here was modelled as $\beta$-cristobalite $\left(\begin{array}{lll}1 & 1 & 1\end{array}\right)$, whose surface non-bridging oxygen atoms were fully protonated, as described elsewhere. ${ }^{12}$ Because quartz (made up by $\mathrm{SiO}_{4}$ tetrahedral structure) is an abundant mineral in earth, the cristobalite crystal with fully protonated non-bridging oxygen atoms is considered a reasonable proxy for hydrophilic rock pore surfaces. ${ }^{13}$ Approximately, 1 n-octane molecule is $12.8 \AA$ in length and 1 carbon dioxide is $5.4 \AA$. We chose to simulate a slit-shaped pore of width slightly larger than these dimensions, $1.9 \mathrm{~nm}$ (determined by the distance between the planes determined by the oxygen atoms of the hydroxyl groups across the pore volume), so that the results will differ substantially compared to those attainable in bulk systems because of surface effects. The simulation box was of size $10.48 \times 10.08 \times 5.19 \mathrm{~nm}^{3}$. The effective pore volume was estimated in $\sim 200.15 \mathrm{~nm}^{3}{ }^{8}, 14$ Periodic boundary conditions were applied in all directions. Because of periodic boundary conditions, the systems considered are composed by silica slabs that are infinitely long along the $\mathrm{X}$ and $\mathrm{Y}$ directions, and separated along the $\mathrm{Z}$ direction by the slitshaped pore. The solid substrate bears no net charge, and all the non-bridging $\mathrm{O}$ atoms in the solid are protonated, yielding a high density of surface - $\mathrm{OH}$ groups. To prevent interactions among fluid molecules among self-replicating images along the $\mathrm{Z}$ direction the solid substrate was of $33 \AA$ in thickness, which is more than double the cut-off distance, set at $14 \AA$.

The total system energy is obtained as the sum of dispersive (van der Waals), electrostatic, bond stretch, bond angle, and dihedral interactions:

$$
E_{\text {total }}=E_{V D W}+E_{\text {electrostatic }}+E_{\text {bond stretch }}+E_{\text {angle bend }}+E_{\text {dihedral }}
$$

$E_{\mathrm{VDW}}$ and $E_{\text {electrostatic }}$ are expressed by 12-6 Lennard-Jones and Coulombic potentials, respectively. Lennard-Jones parameters for non-like components were obtained using Lorentz-Berthelot mixing rules from the values of the pure compounds. ${ }^{15-17}$ The CLAYFF force field ${ }^{18}$ was implemented to simulate silica. $\mathrm{CO}_{2}$ and n-octane were modelled using TraPPE-UA. ${ }^{19}$ The latter force field was chosen for its ability at adequately describing phase coexistence curves. Accurate experimental data are needed to assess whether the combination of CLAYFF and TraPPE-UA force fields yield realistic predictions of fluid behaviour at interfaces. Within this model $\mathrm{CO}_{2}$ is rigid with all atoms on a straight line while octane is a flexible molecule described by bond stretching, angle bending, and dihedral constraints. 
Methyl $\left(\mathrm{CH}_{3}\right)$ and ethyl $\left(\mathrm{CH}_{2}\right)$ groups of $\mathrm{n}$ - octane are treated within the united-atom formalism. The hydrocarbon does not bear partial charges. All atoms on the solid silica, except for $\mathrm{H}$ of the surface $-\mathrm{OH}$ groups, remain rigid throughout the whole length of the simulations.

All the algorithms used to calculate the results reported here are described in our previous work. ${ }^{20}$ The diffusivity of the fluids within the pore is considered as a 2-dimensional translation along the $\mathrm{X}$ and $\mathrm{Y}$ directions, because the movement along the $\mathrm{Z}$ direction is constrained by the confining pore surfaces. To obtain the planar self-diffusion coefficient $D_{\mathrm{s}}$ we calculated the mean square displacement (MSD) following established procedures. ${ }^{8}$ To investigate the effect of $\mathrm{CO}_{2}$ loading on the mobility of confined fluids, 7 different systems were simulated whose details can be found in Table 1. As the molecular density increases, the pressure in the pore is also expected to increase. No attempt was made to compute the pressure representative of the various systems. No bulk was present. All simulations were conducted at $300 \mathrm{~K}$, near the critical temperature for $\mathrm{CO}_{2}$. A schematic representation of a simulated system with 448 carbon dioxide and 282 octane molecules is shown in Figure 1.

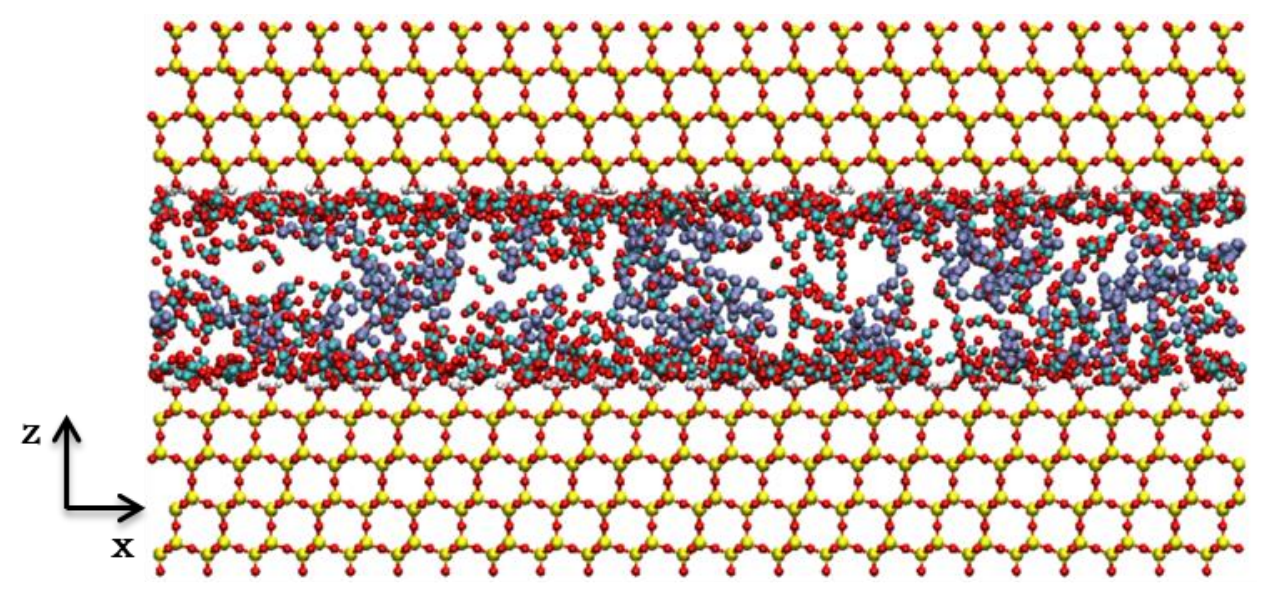

Figure 1. Schematic representation of a simulated system. The solid silica slabs are continuous along both $\mathrm{X}$ and $\mathrm{Y}$ directions, and separated along $\mathrm{Z}$ direction. No bulk region exists. Purple spheres are $\mathrm{CH}_{2}$ and $\mathrm{CH}_{3}$ groups in octane, cyan is $\mathrm{C}$ in carbon dioxide, red is $\mathrm{O}$, white is $\mathrm{H}$, and yellow is $\mathrm{Si}$. 
Table 1. In-plane self-diffusion coefficients calculated for n-octane and $\mathrm{CO}_{2}$ at $300 \mathrm{~K}$ within the silica pore of width $1.9 \mathrm{~nm}$ considered in this study.

\begin{tabular}{cccc}
\hline \multicolumn{4}{c}{ Calculated Self-Diffusion Coefficients $\left(\mathbf{1 0}^{-\mathbf{9}} \mathbf{~ m}^{\mathbf{2}} \mathbf{s}\right)$} \\
\hline N-Octane Molecules & Carbon Dioxide Molecules & $\mathrm{D}_{\text {s-N-Octane }}$ & $\mathrm{D}_{\text {s-Carbon Dioxide }}$ \\
\hline & - & $5.7 \pm 0.2$ & - \\
225 & $10 \pm 1$ & $1.4 \pm 0.5$ \\
282 & 448 & $11.5 \pm 0.5$ & $2.7 \pm 0.5$ \\
& 675 & $11 \pm 1$ & $3.8 \pm 0.5$ \\
& 900 & $10 \pm 0.5$ & $5.2 \pm 0.6$ \\
& 1100 & $7 \pm 0.5$ & $5.8 \pm 0.4$ \\
\hline 400 & - & $4.5 \pm 0.8$ & - \\
\hline
\end{tabular}

To investigate the effect of the pore morphology to the confined species, we conducted an additional simulation in which we modified the morphology of the pore by inserting two step edges (hence creating a structured pore with a trench on its surface), shown in Figure 2, while keeping the total pore volume approximately constant at $\sim 200.15 \mathrm{~nm}^{3}$. The resultant pore is referred to as structured in the remainder of the text. The number of surface $-\mathrm{OH}$ groups and their surface densities are the same in the two pore models (pristine and structured). The fluid simulated in the structured pore was composed of 282 n-octane and 900 $\mathrm{CO}_{2}$ molecules. 


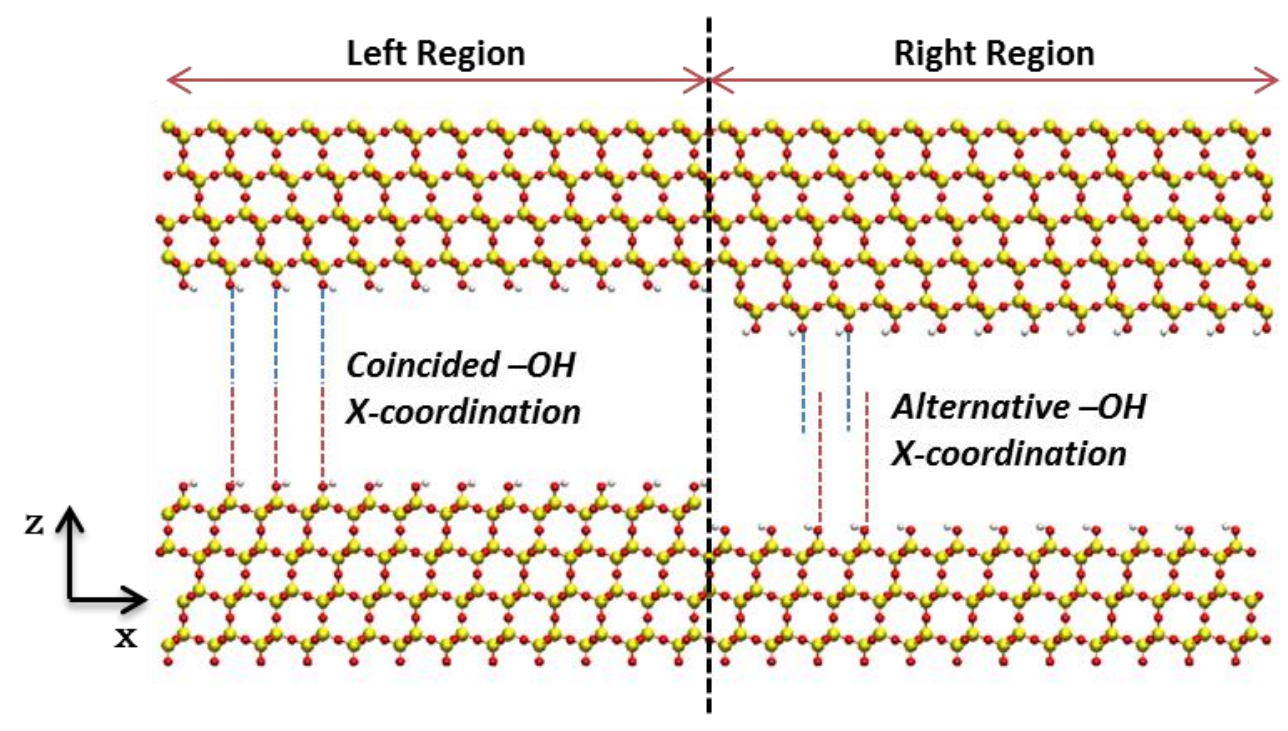

Figure 2. Snapshot representing the structured pore with two step-edges yielding a wide trench (when periodic boundary conditions are considered). The color scheme for the solid substrate is the same as that of Figure 1.

All simulations were carried out within orthorhombic simulation boxes containing a constant number of molecules at fixed volume and constant $T$. $T$ of silica and fluid were controlled separately by two Nosé-Hoover thermostats ${ }^{21,22}$ with relaxation times of 200 fs each. Corrections for long-range electrostatic interactions were taken into account by the particlemesh Ewald summation. ${ }^{23}$ The cut-off distance for all interactions was set at $14 \AA$. The simulations were conducted using the Groningen Machine for Chemical Simulations (GROMACS) simulation package, version 4.5.5. ${ }^{24,} 25$ The leapfrog algorithm ${ }^{26}$ with time steps of $1 \mathrm{fs}$ was implemented. Simulations were conducted for $100 \mathrm{~ns}$ of simulation time for all systems investigated. The simulation time for each system was in the range 40-150 ns, depending on loading. Data analysis was carried out over the last $10 \mathrm{~ns}$ of each simulation. All results were calculated considering the center of mass (COM) of the fluid molecules. 


\section{RESULTS AND DISCUSSION}

\section{Effect of increasing $\mathrm{CO}_{2}$ loading on}

\section{a. Molecular density profiles}

The density profiles in the direction perpendicular to the pore surface are shown in Figure 3. For every curve, there are two distinct peaks with equal heights (symmetric with respect to the pore centre). Panel (a) shows n-octane atomic density profiles in all simulated systems. The results indicate that the locations of the peaks are shifted closer to the pore centre as the number of $\mathrm{CO}_{2}$ increases. For pure n-octane and n-octane in the system with highest $\mathrm{CO}_{2}$ loading considered, there are multiple peaks, which suggest the presence of multiple layers of fluid within the pore. Panels (b)-(f) show the density profiles of both fluids. $\mathrm{CO}_{2}$ shows density peaks closer to the pore walls compared to n-octane, indicating stronger silica surface- $\mathrm{CO}_{2}$ attractions. Visualization of the simulation snapshot in panel (g) confirms the strong association between $\mathrm{CO}_{2}$ molecules and the $-\mathrm{OH}$ groups on the surfaces. This is expected, as $\mathrm{CO}_{2}$ molecules can form hydrogen bonds with the $-\mathrm{OH}$ groups. ${ }^{27,}{ }^{28}$ Note that as its concentration rises, $\mathrm{CO}_{2}$ can also be found near the pore centre. The strong association between $\mathrm{CO}_{2}$ and the pore surface is consistent with recent results reported by Rother et al. for $\mathrm{CO}_{2}$ in porous silica aerogel. ${ }^{29}$ 

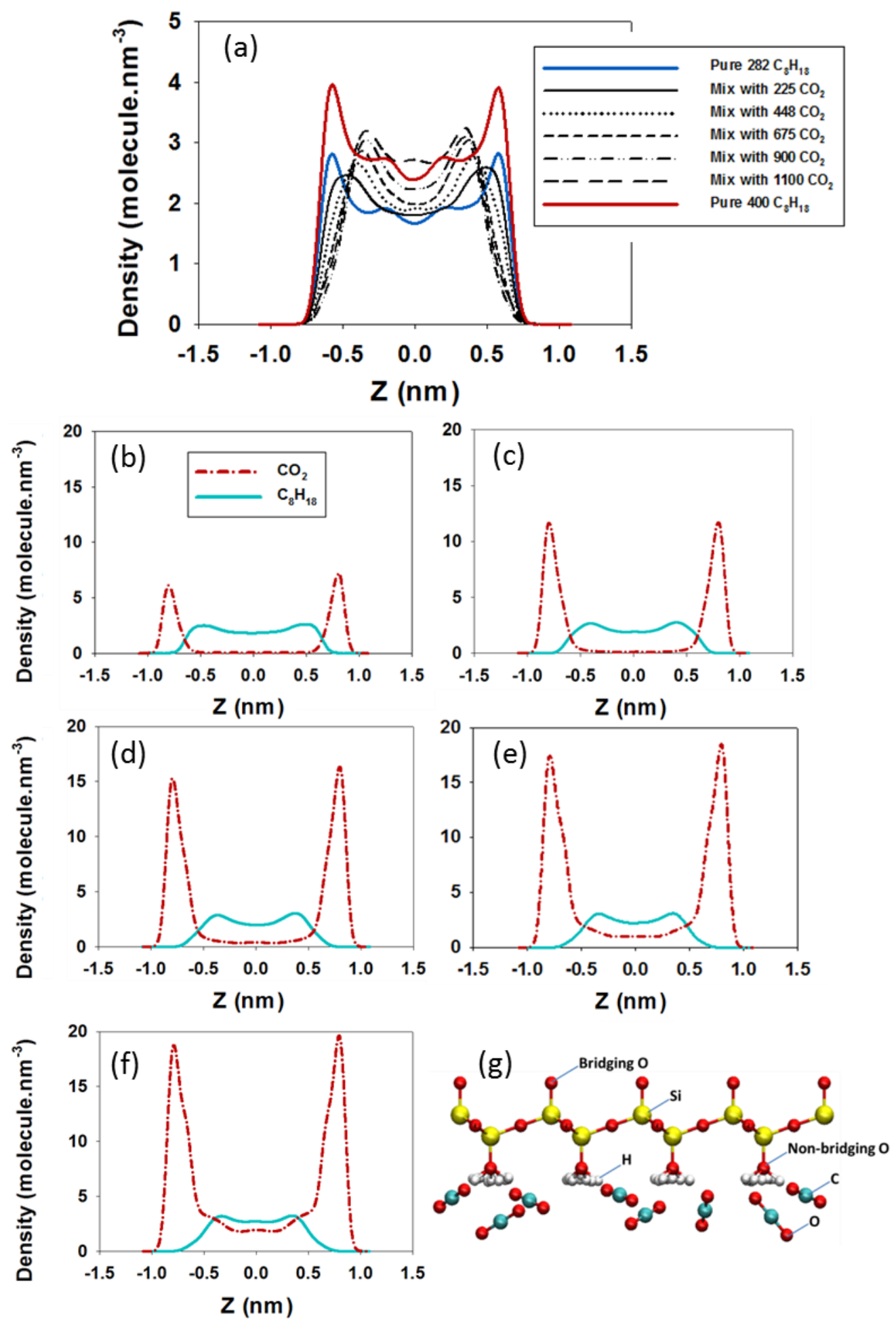

Figure 3. (a) N-octane atomic density profiles in all simulated systems; (b), (c), (d), (e) and (f) are the atomic density profiles obtained for systems composed of $282 \mathrm{n}$-octane and 225 , $448,675,900$ or $1100 \mathrm{CO}_{2}$ molecules at $300 \mathrm{~K}$, respectively. $\mathrm{Z}$ is measured along the direction perpendicular to the pore surface, with $\mathrm{Z}=0$ corresponding to the pore centre; $(\mathrm{g})$ Simulation snapshot for selected $\mathrm{CO}_{2}$ molecules at the interface. Only a few atomic surface silica and hydroxyl groups are shown for clarity. 


\section{b. Orientation of adsorbed n-octane}
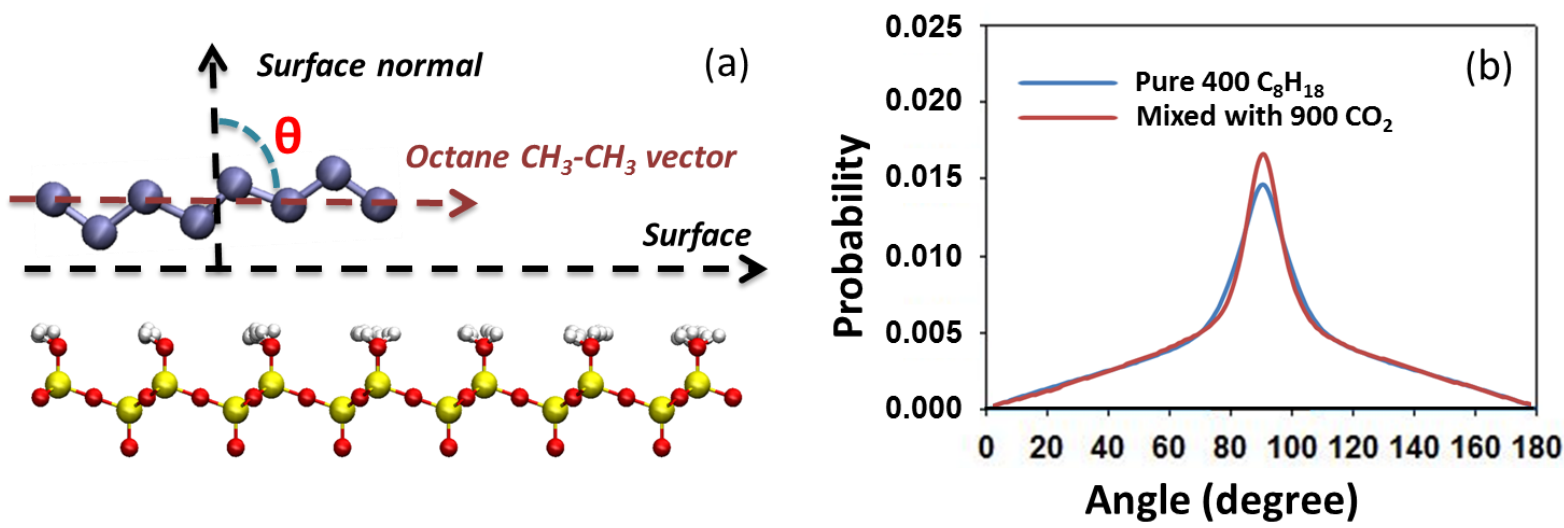

Figure 4. (a) Schematic for the orientation of one adsorbed n-octane molecule. The color scheme for the solid substrate and n-octane model is the same as that of Figure 1; (b) Probability density distribution for the angle $\theta$ for n-octane molecules adsorbed within the first layer adsorbed silica pore in the system composed solely of 400 n-octane (blue line), and for the mixed one composed of $900 \mathrm{CO}_{2}$ and 282 n-octane molecules (red line).

In Figure 4 we report the preferential orientation of adsorbed n-octane molecules. The molecules considered are those within the first adsorbed layer as identified by the density profiles shown in Figure 3. We quantified the probability distribution of the angle $\theta$ formed between the octane $\mathrm{CH}_{3}-\mathrm{CH}_{3}$ vector and the surface normal. When $\theta$ is $0^{\circ}$ or $180^{\circ}$, the molecule is perpendicular to the surface; when $\theta$ is $90^{\circ}$, octane lays parallel to the surface. An illustration is provided in Figure 4, panel (a). The results obtained for the system comprised solely of 400 pure n-octane (blue line), and for the mixed one composed of $900 \mathrm{CO}_{2}$ and 282 n-octane molecules (red line) are shown in panel (b). Data for other binary mixtures are not shown for brevity. The results show that octane molecules within the first adsorbed layer preferentially orient parallel to the pore surface. Similar calculations were conducted for $\mathrm{CO}_{2}$ in our recent work. ${ }^{11}$ Those results show that each adsorbed $\mathrm{CO}_{2}$ molecule tends to interact simultaneously with more than 1 surface $-\mathrm{OH}$ at a time.

\section{c. Diffusivities}

The results for the self-diffusion coefficients for both $\mathrm{CO}_{2}$ and n-octane are summarized in Table 1. The pore surface is symmetric and periodic in $\mathrm{X}$ and Y. Hence, diffusion is expected to be isotropic along $\mathrm{X}$ and $\mathrm{Y}$ directions. No effort was made to confirm this. In general, we observe a non-monotonic change in n-octane diffusivity as a function of $\mathrm{CO}_{2}$ loading. At low 
to medium loadings, n-octane diffusivity increases with $\mathrm{CO}_{2}$ loading. Based on the density profiles, this increase is associated with the displacement of n-octane from the pore surfaces due to the preferential adsorption of $\mathrm{CO}_{2}$. These observations are qualitatively consistent with those recently reported for $\mathrm{CO}_{2}$-butane mixtures confined in a silica pore similar to the one considered here. Those results, obtained at different temperatures, showed a clear reduction in the activation energy for hydrocarbon diffusion due to the presence of $\mathrm{CO}_{2}$. The results presented herein are consistent with experimental data reported by Vidoni, ${ }^{30}$ who reported a reduction in the experimentally measured activation energy for $\mathrm{CH}_{4}$ diffusion in DD3R materials due to the co-adsorption of $\mathrm{CO}_{2}$. At high $\mathrm{CO}_{2}$ loadings our results show a drop in $\mathrm{n}$ octane self-diffusion coefficient, likely because of pore crowding (i.e., more frequent molecular collisions).

One system consisting of 400 n-octane molecules and no $\mathrm{CO}_{2}$ was simulated to confirm that n-octane enhanced diffusivity is due to $\mathrm{CO}_{2}$. Indeed, the results show that the n-octane selfdiffusion coefficient decreases as its density increases, confirming that $\mathrm{CO}_{2}$ is essential for enhancing the diffusion of n-octane within the systems considered here. To further confirm this conclusion, we conducted an additional simulation for which the pore size was reduced by $4.8 \AA$ so that the removed pore volume approximately equals the volume occupied by $1100 \mathrm{CO}_{2}$ in the mixture. The reduced pore size was estimated by the shift in distance for octane density profile peaks with and without $1100 \mathrm{CO}_{2}$ molecules (see Figure 3a). 282 pure n-octane molecules were simulated for $40 \mathrm{~ns}$ and the calculated n-octane self-diffusion coefficient was $5.2 \times 10^{-9} \mathrm{~m}^{2} / \mathrm{s}$, which was lower than any values obtained for $282 \mathrm{n}$-octane in the presence of $\mathrm{CO}_{2}$ (see Table $\mathbf{1}$ ).

In contrasts to the results obtained for n-octane, our results show that the self-diffusion coefficient for $\mathrm{CO}_{2}$ monotonically increases with its loading. This is qualitatively consistent with experimental data reported by Wang et al. ${ }^{31}$ for octane and decane in microporous BPL activated carbons, and it is due to the presence of high-energy adsorption sites. In our systems, $\mathrm{CO}_{2}$ molecules preferentially adsorb on the surface $-\mathrm{OH}$ groups. It was expected that the averaged self-diffusion coefficient for $\mathrm{CO}_{2}$ remains low until all the available preferential adsorption sites are occupied. While this expectation is met qualitatively, the details vary. Analysis of the simulation snapshots suggests that the pore surfaces are saturated by adsorbed $\mathrm{CO}_{2}$ when the number of molecules present is between 448 and 675. As the simulation cell contains a total of $960-\mathrm{OH}$ groups on the 2 pore walls, our results suggest 
that, on average, one $\mathrm{CO}_{2}$ molecule adsorbs on $2-\mathrm{OH}$ groups. The results shown in Table 1 suggest that the diffusivity of $\mathrm{CO}_{2}$ increases even before the two surfaces are saturated.

By analysing the trajectories of representative $\mathrm{CO}_{2}$ molecules (shown in Figure 5 below) we can assess the mechanism responsible for this observation. When only $225 \mathrm{CO}_{2}$ molecules are present (Figure 5a), the trajectories show little mobility along the X-Y plane, and rarely the $\mathrm{CO}_{2}$ molecules desorb from the silica surfaces. The situation begins to differ when 448 molecules are present (Figure 5b). Although the $\mathrm{CO}_{2}$ molecules still do not desorb from the silica surface, a noticeable amount of small density fluctuations are observed near the surface, possibly caused by a competition between different $\mathrm{CO}_{2}$ molecules to adsorb on the $-\mathrm{OH}$ groups. These fluctuations allow the $\mathrm{CO}_{2}$ molecules to escape the attractive wells near the surface, and the molecular diffusion along the $\mathrm{X}-\mathrm{Y}$ pane increases. The behaviour just described persists when the number of $\mathrm{CO}_{2}$ molecules increases to 675 (Figure 5c), but exhibits an additional event wherein, occasionally, the $\mathrm{CO}_{2}$ molecules desorb from one silica surface and travel across the pore to adsorb on the other surface. While the $\mathrm{CO}_{2}$ molecules are near the pore centre, they can travel far along the X-Y plane, leading to enhanced selfdiffusion coefficients. As more $\mathrm{CO}_{2}$ molecules are present, the likelihood of desorption also increases (see trajectories shown in Figure 5d). Should the density of $\mathrm{CO}_{2}$ simulated within the pore of Figure 1 be increased beyond the range considered herein, it is expected that its self-diffusion coefficient will reach a maximum and then decrease (due to steric hindrance). 

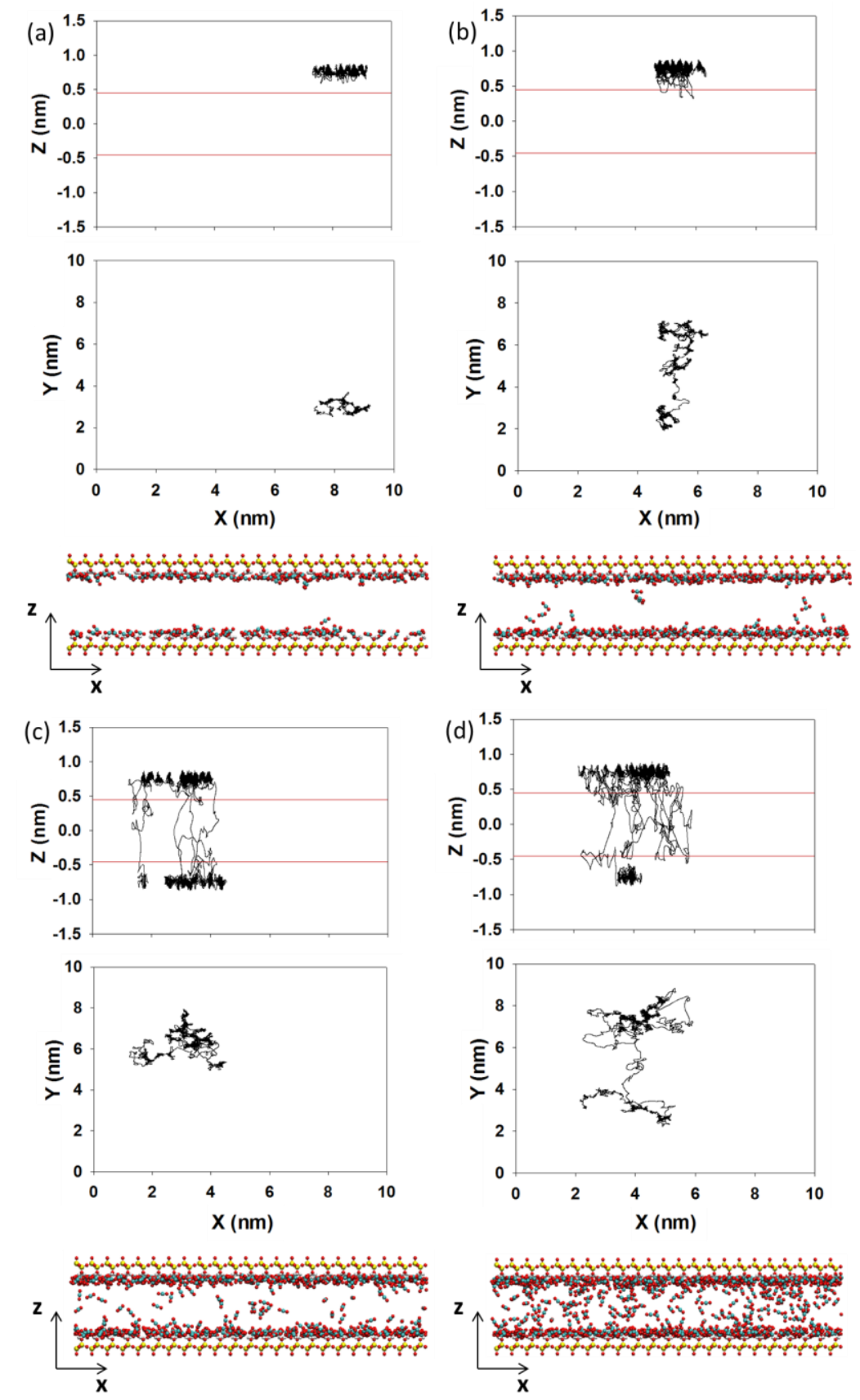

Figure 5. Trajectories and simulation snapshots for mixtures composed of 282 n-octane and (a) 225 , (b) 448 , (c) 675 or (d) $900 \mathrm{CO}_{2}$ molecules at $300 \mathrm{~K}$. $\mathrm{Z}$ is the direction perpendicular to the pore surface, with $\mathrm{Z}=0$ corresponding to the pore centre. The red lines identify the layer of $\mathrm{CO}_{2}$ adsorbed on the pore surfaces (they correspond to the minima in the $\mathrm{CO}_{2}$ density profiles in Figure 1). The black lines reproduce the trajectories of one $\mathrm{CO}_{2}$ molecule in each system for $1 \mathrm{~ns}$. All simulation snapshots display $\mathrm{CO}_{2}$ molecules (octane molecules are not shown for clarity) confined within the silica pore (only the pore surface is shown for clarity). Cyan is $\mathrm{C}$ in $\mathrm{CO}_{2}$, red is $\mathrm{O}$, white is $\mathrm{H}$, and yellow is $\mathrm{Si}$. 


\section{Effect of pore morphology}

In Figure 6, panels (a) and (b), we report simulation snapshots representing the two pore models with the confined $900 \mathrm{CO}_{2}$ molecules at $300 \mathrm{~K}$ (n-octane is not shown for clarity). In panels (c) and (d) we show the corresponding $\mathrm{CO}_{2}$ density profiles along the $\mathrm{X}$-direction. While $\mathrm{CO}_{2}$ in the pore with flat surfaces (pristine pore) displays a rather uniform molecular distribution along the pore length (Figure 6c), it distributes unevenly on the structured pore surface, with somewhat higher molecular density near the steps (Figure 6d). It is worth mentioning that $\mathrm{CO}_{2}$ distributions for the left and right halves of the structured pore are different due to the difference in the $-\mathrm{OH}$ groups distribution on the two pore surfaces facing across the pore volume (see Figure 2). Explicitly, in the left half of the pore, the - $\mathrm{OH}$ groups of the top and bottom surfaces are aligned along the $\mathrm{X}$ coordinate, while in the right half of the pore this does not occur.

The calculated self-diffusion coefficients obtained during the 80 ns simulations within the structured pore were $6.6 \times 10^{-9}$ and $4.8 \times 10^{-9} \mathrm{~m}^{2} / \mathrm{s}$ for n-octane and $\mathrm{CO}_{2}$, respectively. These results show that n-octane molecules diffuse faster in this structured pore in the presence of $\mathrm{CO}_{2}$ than when they are pure in the pristine slit-shaped pore. However, the results also show that $\mathrm{CO}_{2}$ is not as effective in enhancing n-octane mobility as it is in the pristine slit-shaped pore. In fact, the self-diffusion coefficient for n-octane in a fluid mixture of the same composition decreases by about $35 \%$ due to the change in pore morphology. The selfdiffusion coefficient obtained for $\mathrm{CO}_{2}$ slightly decreases compared to data obtained in the pristine pore due to the change in pore geometry, which is due to a slight increase in the pore surface area (which is larger on the structured pore by $\sim 5 \%$ ), and perhaps due to $\mathrm{CO}_{2}$ accumulation near the surface edges. A series of studies of $\mathrm{CO}_{2}-$ octane mixtures confined in different pore substrates (i.e. calcite and muscovite) are being conducted to further investigate the effects of surface chemistry on confined fluids. These results will be presented and discussed in future reports. 


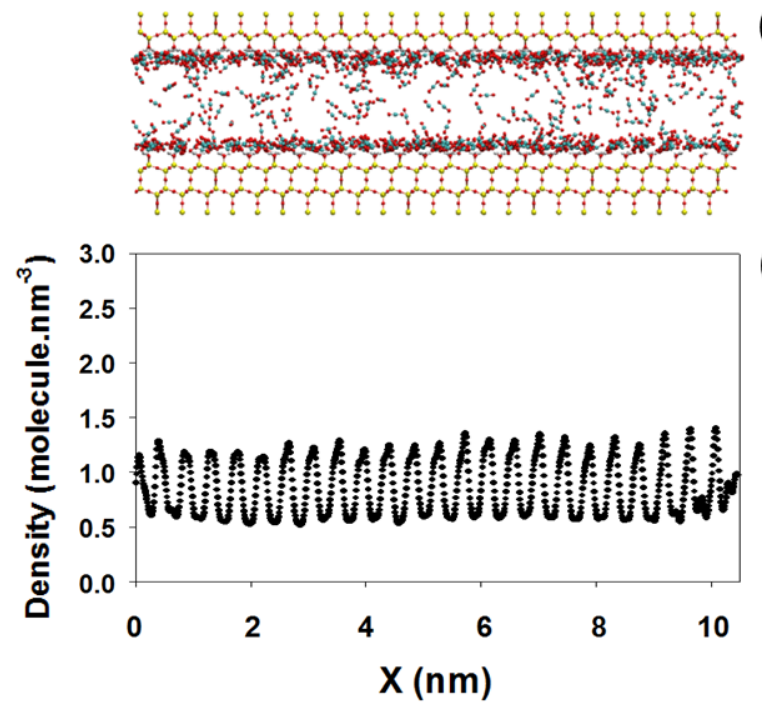

(a)

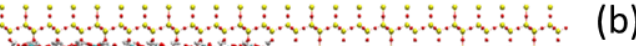

(b)

(c)

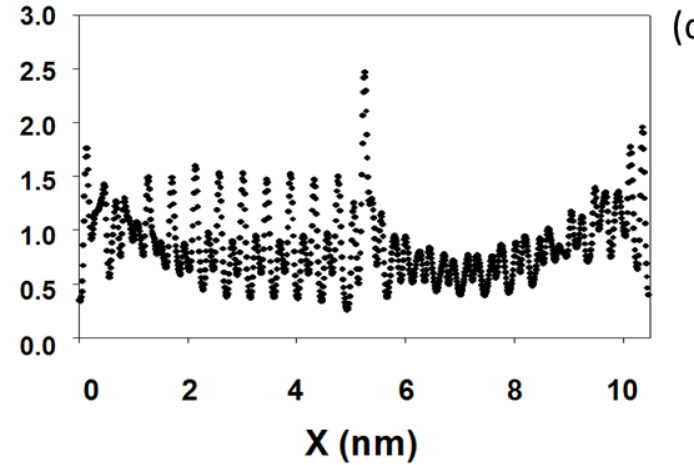

(d)

Figure 6. (a), (b) Representative simulation snapshots of $900 \mathrm{CO}_{2}$ in the pristine and structured pores, respectively, at $300 \mathrm{~K}$ (the 282 n-octane molecules are not shown for clarity). (c), (d) $\mathrm{CO}_{2}$ molecular density profiles along the X-direction of the two pore models.

The colour code is the same as that used in Figure 1.

\section{CONCLUSIONS}

We conducted equilibrium molecular dynamics simulations for pure n-octane confined within a slit-shaped silica pore of width $1.9 \mathrm{~nm}$. The simulations were conducted at $300 \mathrm{~K}$ for a fixed amount of n-octane and increasing number of $\mathrm{CO}_{2}$ molecules. The silica surfaces were obtained from $\beta$-cristobalite and were fully protonated. The results show that $\mathrm{CO}_{2}$ preferentially adsorbs on the silica surface, displacing n-octane towards the pore centre. The n-octane molecules have a preferential parallel orientation with respect to the pore surface and this orientation is enhanced with the presence of $\mathrm{CO}_{2}$. The capacity for $\mathrm{CO}_{2}$ is of approximately $1 \mathrm{CO}_{2}$ molecule every two $-\mathrm{OH}$ groups on our surfaces. When $\mathrm{CO}_{2}$ loading exceeds this value, $\mathrm{CO}_{2}$ can be found near the pore centre. Our results show that the selfdiffusion coefficient for n-octane increases as $\mathrm{CO}_{2}$ is added to the system, but eventually reaches a maximum and then decreases because of steric hindrance. Sample simulations conducted with higher loading of n-octane and no $\mathrm{CO}_{2}$ present, as well as test simulations conduced for pure n-octane in narrower pores confirm that the results just discussed are due to the preferential adsorption of $\mathrm{CO}_{2}$ on the pore surfaces, which in this way acts as an effective molecular lubricant for n-octane. Within the conditions considered (up to $1100 \mathrm{CO}_{2}$ molecules), our results show that the self-diffusion coefficient for $\mathrm{CO}_{2}$ increases 
monotonically with its loading. Although this is consistent with the presence of preferential adsorption sites on the pore surface, analysis of the simulation trajectories suggests that even at loadings lower than full $\mathrm{CO}_{2}$ adsorption capacity can lead to density fluctuations near the pore surfaces, which lead to higher self-diffusion coefficients.

Although the simulations presented here are only conducted for slit-shaped silica pores of one width with a model hydrocarbon chain at one temperature, the results help to complete our understanding about the molecular phenomena related to adsorption and transport of $\mathrm{CO}_{2}-$ hydrocarbon mixtures in sub-surface environments, in addition to those found in our previous works. Additional test simulations, conducted for a pore of equal volume to the slit-shaped pore considered above, but with step edges present on the surface, suggest that the pore morphology, and in particular the presence of edges, has a strong effect on the fluid behaviour under confinement.

\section{ACKNOWLEDGEMENTS}

Generous allocations of computing time were provided by the University College London Research Computing Platforms Support (LEGION), the Oklahoma Supercomputing Center for Education and Research (OSCER) and the National Energy Research Scientific Computing Center (NERSC) at Lawrence Berkeley National Laboratory. NERSC is supported by the DOE Office of Science under Contract No. DE-AC02-05CH11231. Ms. Thu Le wishes to thank financial support from the Sloan Foundation, via the Deep Carbon Observatory. DRC was supported by the Department of Energy, Office of Basic Energy Sciences, Geosciences program under grant DE-SC0006878. AS acknowledges partial financial support from a Marie Curie Career Initiation Grant. 


\section{References}

1. Khosravi, M.; Bahramian, A.; Emadi, M.; Rostami, B.; Roayaie, E., Mechanistic investigation of bypassed-oil recovery during $\mathrm{CO} 2$ injection in matrix and fracture. Fuel 2014, 117, Part A, (0), 43-49.

2. Gislason, S. R.; Oelkers, E. H., Carbon Storage in Basalt. Science 2014, 344, (6182), 373-374.

3. Himeno, S.; Komatsu, T.; Fujita, S., High-Pressure Adsorption Equilibria of Methane and Carbon Dioxide on Several Activated Carbons. J. Chem. Eng. Data 2005, 50, (2), 369-376.

4. Huang, L.; Zhang, L.; Shao, Q.; Lu, L.; Lu, X.; Jiang, S.; Shen, W., Simulations of Binary Mixture Adsorption of Carbon Dioxide and Methane in Carbon Nanotubes: Temperature, Pressure, and Pore Size Effects. J. Phys Chem. C 2007, 111, (32), 11912-11920.

5. Bleken, B.-T. L.; Lillerud, K. P.; Splith, T.; Pusch, A.-K.; Stallmach, F., PFG NMR diffusion measurements of $\mathrm{CH} 4$ and $\mathrm{CO} 2$ through large ZSM-58-crystals. Microporous Mesoporous Mater. 2013, 182, (0), 25-31.

6. Cole, D. R.; Ok, S.; Striolo, A.; Phan, A., Hydrocarbon Behavior at Nanoscale Interfaces. Reviews in Mineralogy and Geochemistry 2013, 75, (1), 495-545.

7. Cole, D. R.; Ok, S.; Phan, A.; Rother, G.; Striolo, A.; Vlcek, L., Carbon-Bearing Fluids at Nanoscale Interfaces. Procedia Earth and Planetary Science 2013, 7, (0), 175-178.

8. Le, T.; Striolo, A.; Cole, D. R., Propane simulated in silica pores: Adsorption isotherms, molecular structure, and mobility. Chem. Eng. Sci. 2015, 121, (0), 292-299.

9. Gruszkiewicz, M. S.; Rother, G.; Wesolowski, D. J.; Cole, D. R.; Wallacher, D., Direct Measurements of Pore Fluid Density by Vibrating Tube Densimetry. Langmuir 2012, 28, (11), 50705078.

10. Rother, G.; Melnichenko, Y. B.; Cole, D. R.; Frielinghaus, H.; Wignall, G. D., Microstructural Characterization of Adsorption and Depletion Regimes of Supercritical Fluids in Nanoporest. The Journal of Physical Chemistry C 2007, 111, (43), 15736-15742.

11. Le, T.; Striolo, A.; Cole, D. R., CO2-C4H10 Mixtures Simulated in Silica Slit Pores: Relation between Structure and Dynamics. J. Phys Chem. C 2015, 119, (27), 15274-15284.

12. Argyris, D.; Tummala, N. R.; Striolo, A.; Cole, D. R., Molecular Structure and Dynamics in Thin Water Films at the Silica and Graphite Surfaces. The Journal of Physical Chemistry C 2008, 112, (35), 13587-13599.

13. Rother, G.; Krukowski, E. G.; Wallacher, D.; Grimm, N.; Bodnar, R. J.; Cole, D. R., Pore Size Effects on the Sorption of Supercritical CO2 in Mesoporous CPG-10 Silica. J. Phys Chem. C 2011, 116, (1), 917-922.

14. Kaneko, K.; Cracknell, R. F.; Nicholson, D., Nitrogen Adsorption in Slit Pores at Ambient Temperatures: Comparison of Simulation and Experiment. Langmuir 1994, 10, (12), 4606-4609.

15. Allen, M. P.; Tildesley, D. J., Computer simulation of liquids. Clarendon Pr.: Oxford, 2004.

16. Berthelot, D., Sur le mélange des gaz. Comptes rendus hebdomadaires des séances de l'Académie des Sciences 1898, (126), 1703-1855.

17. Lorentz, H. A., Ueber die Anwendung des Satzes vom Virial in der kinetischen Theorie der Gase. Annalen der Physik 1881, 248, (1), 127-136.

18. Cygan, R. T.; Liang, J.-J.; Kalinichev, A. G., Molecular Models of Hydroxide, Oxyhydroxide, and Clay Phases and the Development of a General Force Field. J. Phys Chem. B 2004, 108, (4), 12551266.

19. Martin, M. G.; Siepmann, J. I., Transferable Potentials for Phase Equilibria. 1. United-Atom Description of n-Alkanes. J. Phys Chem. B 1998, 102, (14), 2569-2577.

20. Ho, T. A.; Argyris, D.; Cole, D. R.; Striolo, A., Aqueous $\mathrm{NaCl}$ and $\mathrm{CsCl}$ Solutions Confined in Crystalline Slit-Shaped Silica Nanopores of Varying Degree of Protonation. Langmuir 2011, 28, (2), 1256-1266.

21. Hoover, W. G., Canonical dynamics: Equilibrium phase-space distributions. Phys. Rev. A 1985, 31, (3), 1695-1697. 
22. Nosé, S., A molecular dynamics method for simulations in the canonical ensemble. Mol. Phys. 1984, 52, (2), 255-268.

23. Essmann, U.; Perera, L.; Berkowitz, M. L.; Darden, T.; Lee, H.; Pedersen, L. G., A smooth particle mesh Ewald method. J. Chem. Phys 1995, 103, (19), 8577-8593.

24. Hess, B.; Kutzner, C.; van der Spoel, D.; Lindahl, E., GROMACS 4: Algorithms for Highly Efficient, Load-Balanced, and Scalable Molecular Simulation. J. Chem. Theory Comput. 2008, 4, (3), 435-447.

25. Van Der Spoel, D.; Lindahl, E.; Hess, B.; Groenhof, G.; Mark, A. E.; Berendsen, H. J. C., GROMACS: Fast, flexible, and free. J. Comput. Chem. 2005, 26, (16), 1701-1718.

26. Hockney, R. W.; Goel, S. P.; Eastwood, J. W., Quiet high-resolution computer models of a plasma. J. Comput. Phys. 1974, 14, (2), 148-158.

27. Arunan, E.; Desiraju, G. R.; Klein, R. A.; Sadlej, J.; Scheiner, S.; Alkorta, I.; Clary, D. C.; Crabtree, R. H.; Dannenberg, J. J.; Hobza, P.; Kjaergaard, H. G.; Legon, A. C.; Mennucci, B.; Nesbitt, D. J., Definition of the hydrogen bond (IUPAC Recommendations 2011). Pure Appl. Chem. 2011, 83, (8), 1637-1641.

28. Sato, H.; Matubayasi, N.; Nakahara, M.; Hirata, F., Which carbon oxide is more soluble? Ab initio study on carbon monoxide and dioxide in aqueous solution. Chem. Phys. Lett. 2000, 323, (3-4), 257-262.

29. Rother, G.; Vlcek, L.; Gruszkiewicz, M. S.; Chialvo, A. A.; Anovitz, L. M.; Bañuelos, J. L.; Wallacher, D.; Grimm, N.; Cole, D. R., Sorption Phase of Supercritical CO2 in Silica Aerogel: Experiments and Mesoscale Computer Simulations. J. Phys Chem. C 2014, 118, (28), 15525-15533.

30. Vidoni, A. Adsorption and Diffusion of Light Hydrocarbon in DDR Zeolite. The University of Maine 2011.

31. Wang, Y.; Mahle, J. J.; Furtado, A. M. B.; Glover, T. G.; Buchanan, J. H.; Peterson, G. W.; LeVan, M. D., Mass Transfer and Adsorption Equilibrium for Low Volatility Alkanes in BPL Activated Carbon. Langmuir 2013, 29, (9), 2935-2945. 